\title{
Importância dos minerais na alimentação do pré-termo extremo
}

\author{
Minerals in the nutrition of extremely low birth weight infants
}

Cleide E. P. Trindade*

\section{Resumo}

Objetivo: Revisão crítica da literatura sobre os minerais cálcio, fósforo e microelementos na nutrição do pré-termo extremo, considerando a importância no crescimento, mineralização óssea e como componente de dietas.

Fontes dos dados: Utilizamos o banco de dados MEDLINE e o Cochrane Database of Systematic Reviews, de 1994 a 2004. Selecionamos artigos com enfoques originais, artigos de revisão e livros específicos.

Síntese dos dados: As dificuldades na nutrição de prematuros extremos aumentam a freqüência de prematuros com restrição de crescimento, cujas conseqüências futuras estão para serem determinadas. Todavia, há pouca literatura sobre minerais, especialmente sobre micronutrientes na nutrição do pré-termo extremo, considerando-se o deficiente armazenamento e a importância destes em nutrição. O principal enfoque desta revisão foi sobre o cálcio e o fósforo na mineralização óssea e na suplementação em nutrição parenteral e enteral, bem como a avaliação crítica da nutrição pós-alta sobre a mineralização óssea. São abordadas as necessidades de micronutrientes, principalmente selênio e zinco, e a função antioxidante do selênio na provável prevenção de doenças do prematuro com atuação de radicais livres.

Conclusão: Considerando os baixos estoques de minerais em prematuros extremos, há necessidade de mais pesquisas sobre minerais na nutrição destes prematuros para definir suas reais necessidades, os aspectos metabólicos, bem como aplicar os conhecimentos na formulação de dietas que permitam prevenir quadros de deficiência e conseqüências a longo prazo. Ainda há controvérsias sobre a influência de fórmulas pós-alta sobre a evolução da doença óssea da prematuridade.

J Pediatr (Rio J). 2005;81(1 Supl):S43-S51: Prematuro, minerais, cálcio, selênio, micronutrientes, nutrição.

\section{Introdução}

São denominados prematuros extremos ou prematuros de extremo baixo-peso aqueles recém-nascidos com peso de nascimento inferior a $1.000 \mathrm{~g}$. Estes recém-nascidos apresentam extrema imaturidade e, portanto, a nutrição

* Professora titular de Pediatria, Faculdade de Medicina de Botucatu, Universidade Estadual Paulista (UNESP), Botucatu, SP.

Como citar este artigo: Trindade CE. Importância dos minerais na alimentação do pré-termo extremo. J Pediatr (Rio J). 2005;81(1 Supl):S43-S51.

\section{Abstract}

Objective: To review the literature on the role of calcium, phosphorus and trace elements in the nutrition of extremely low birth weight infants, considering their importance for metabolism, bone mineralization and as dietary components.

Sources of data: MEDLINE, the Cochrane Database of Systematic Reviews and books on nutrition were searched between 1994 and 2004. Original research studies and reviews were selected.

Summary of the findings: Extremely preterm infants are frequently growth-restricted at hospital discharge as a consequence of difficulties in the provision of adequate nutrition. The long-term effects of this growth restriction need to be determined. There is a paucity of studies about the role of minerals, especially micronutrients, in the nutrition of extremely preterm infants. The principal focus of this review was on calcium and phosphorus metabolism, bone mineralization and parenteral and enteral supplementation. A critical evaluation of post-discharge nutrition and its influence upon growth and bone mineralization was presented. Selenium and zinc requirements and the role of selenium as an antioxidant with possible effects on free radical diseases of the preterm infant were discussed.

Conclusions: Extremely preterm infants have low mineral reserves and, as a consequence, may have deficiencies in the postnatal period if they do not receive parenteral or enteral supplementation. More studies are needed to elucidate the actual requirements and the appropriate supplementation of micronutrients. There are controversies about the outcome and the influence of postdischarge nutrition on bone disease of prematurity.

J Pediatr (Rio J). 2005;81(1 Supl):S43-S51: Infant, preterm, minerals, calcium, selenium, micronutrients, nutrition.

deve ser ajustada a cada circunstância e não apenas adaptada ao peso de nascimento. Não há definições precisas de suas necessidades nutricionais, e, assim, devem ser monitorizados com freqüência quanto ao crescimento e repercussões metabólicas da nutrição.

Com os avanços nos cuidados ministrados aos prematuros, cada vez mais sobrevivem recém-nascidos com peso muito baixo e pouca idade gestacional. Estes prematuros têm características metabólicas próprias e grande número de doenças e complicações no período pós-natal, tornando 
sua nutrição um verdadeiro desafio. Atualmente, é dada grande importância à nutrição pós-natal destes prematuros, pois a desnutrição fetal e, possivelmente, a desnutrição pós-natal têm sido consideradas, em trabalhos epidemiológicos, importantes na determinação de doenças do adulto, tais como: diabetes, hipertensão, cardiopatias. Portanto, aumenta a responsabilidade do neonatologista em propiciar a estes recém-nascidos uma nutrição que Ihes garanta crescimento físico e desenvolvimento neuropsicomotor adequados. Dentro desta perspectiva, a nutrição de prematuros extremos com foco nos minerais adquire importância, pois os minerais apresentam funções diferenciadas no organismo, embora haja pouco conhecimento sobre os mesmos, principalmente no que se refere à nutrição de prematuros extremos. Tendo em vista que alguns são pouco estudados, cabe ressaltar que, entre os minerais, o cálcio e o fósforo são os mais abordados na literatura, em função de distúrbios metabólicos agudos no período neonatal imediato, como a hipocalcemia neonatal precoce, bem como manifestações tardias, como a doença óssea metabólica do prematuro. Entretanto, vários minerais, como o zinco, o magnésio, o ferro, o selênio e outros microelementos, também são importantes constituintes da nutrição de prematuros, embora para a maioria não haja, na deficiência, manifestações clínicas evidentes.

\section{Cálcio e fósforo}

O cálcio ( $C a$ ) e o fósforo $(P)$ são importantes para a mineralização óssea fetal e pós-natal. O período de maior desenvolvimento esquelético e de mineralização óssea ocorre no terceiro trimestre de gestação, quando o feto incorpora quantidades elevadas de cálcio e fósforo, em média 120 a 140 mg/kg/dia e 60 a 75 mg/kg/dia, respectivamente.

Os níveis de incorporação mineral aumentam exponencialmente entre 24 e 37 semanas de gestação, resultando em $80 \%$ de acúmulo de minerais no terceiro trimestre. Como conseqüência, prematuros nascidos com idades gestacionais entre 24 e 34 semanas ficarão privados do suprimento intra-uterino de cálcio, afetando a mineralização óssea intra-uterina e a pós-natal.

Estimativas das necessidades de cálcio para prematuros são inferidas a partir da composição química de tecidos fetais, calculadas em várias idades gestacionais. Embora haja críticas a esses métodos, há concordância em que os níveis de incorporação variam de $120 \mathrm{mg} / \mathrm{kg} / \mathrm{dia}$ com 26 semanas de idade gestacional a $130-140 \mathrm{mg} / \mathrm{kg} / \mathrm{dia}$ com 36 semanas $^{1}$ e $150-155 \mathrm{mg} / \mathrm{kg} /$ dia entre $36-38$ semanas. A medida do conteúdo mineral ósseo (CMO) de recém-nascidos de várias idades gestacionais, determinada por absorção de fótons, mostra que o conteúdo mineral do terço distal do rádio aumenta de $25 \mathrm{mg} / \mathrm{cm}$ com 26 semanas para $65 \mathrm{mg} / \mathrm{cm}$ com 36 semanas. O CMO e a área do osso (AO), medidos por DXA (dual energy $X$ ray absorciometry), mostraram que ambas as medidas correlacionam-se com o peso corporal, o comprimento e a idade gestacional².
A absorciometria por duplo raio $X$ é considerada $a$ técnica não invasiva mais precisa para definir a mineralização óssea in vivo. Empregando essa técnica e calculando o $\mathrm{CMO}$ e a $\mathrm{AO}$, observou-se que ambas as medidas correlacionam-se com o peso corporal, comprimento e idade gestacional. Em análise multivariada, o peso corporal foi o maior preditor destes parâmetros ${ }^{2}$.

A avaliação da incorporação de Ca na fase fetal tem implicações práticas, uma vez que, na fase pós-natal, um prematuro deve receber cálcio suficiente para incorporação óssea semelhante à obtida na fase intra-uterina. Portanto, na fase pós-natal de prematuros extremos, o objetivo é ministrar nutrição de forma que este possa recuperar os níveis de crescimento e de mineralização intra-uterinos. Para que haja mineralização, deve haver síntese de colágeno da matriz óssea, o que resulta em necessidade de ingestão elevada de proteínas e energia para prematuros de extremo baixo peso. Juntamente com o crescimento do esqueleto, há necessidade de ministração de cálcio e fósforo em quantidades adequadas para que haja mineralização ${ }^{2}$. Em prematuros extremos, alguns fatores contribuem para a dificuldade em manter metas de crescimento e de mineralização, fazendo com que a doença óssea metabólica ocorra em aproximadamente $50 \%$ destes prematuros.

Logo após o nascimento, o cálcio ministrado destina-se a prevenir ou tratar a hipocalcemia neonatal, freqüente em prematuros extremos e decorrente da interrupção do cálcio provindo através da placenta, de níveis elevados de calcitonina que persistem nos primeiros dias de vida e de insuficiente liberação de paratormônio por resposta imatura das glândulas paratireóides ${ }^{3}$. No período pós-natal, ocorrem dificuldades em ministrar maiores quantidades de Ca e $P$ com a nutrição, pois estes pequenos prematuros freqüentemente apresentam intercorrências como a síndrome de desconforto respiratório, a hemorragia peri-intraventricular e a doença pulmonar crônica. Freqüentemente, estão em restrição hídrica, além de apresentar intolerância alimentar, condições estas que reduzem o aporte de proteínas, energia, cálcio e fósforo.

\section{Suprimento de cálcio por nutrição parenteral}

A nutrição parenteral é a maior fonte de nutrição para prematuros extremos nos primeiros dias, até que possam tolerar nutrição enteral e receber quantidades adequadas de nutrientes, suficientes para ocorrer o crescimento. Entretanto, em relação ao cálcio e ao fósforo, há dificuldades em ministrar, através da nutrição parenteral, quantidades suficientes que se equiparem às necessidades intra-uterinas. O fator limitante refere-se à solubilidade do cálcio na presença do fósforo, além da interferência de vários fatores na manutenção da estabilidade das soluções, como: fonte de cálcio, concentração, pH da solução, concentração de glicose, ordem de colocação dos componentes, temperatura da solução.

As soluções mais empregadas consistem em gluconato de cálcio associado a sais de fosfato mono ou dibásico. A solubilidade melhora adicionando-se os sais de fósforo antes dos sais de cálcio e empregando-se maiores concen- 
trações de aminoácidos. Novas formulações, contendo glicerofosfato e fosfato monobásico, permitem fornecer maiores quantidades de cálcio e de fósforo, até $86 \mathrm{mg} / \mathrm{dl}$ de cálcio e $46 \mathrm{mg} / \mathrm{dl}$ de fósforo, concentrações ainda insuficientes para permitir a mineralização óssea adequada ${ }^{2}$.

Koo \& Tsang ${ }^{4}$, em 1993, ao avaliarem diversos estudos sobre balanço metabólico em recém-nascidos estáveis e em parenteral recebendo 58 a $76 \mathrm{mg} / \mathrm{kg} /$ dia de cálcio e 38 a 54 $\mathrm{mg} / \mathrm{kg} /$ dia de fósforo, observaram retenção de $88 \%$ a $94 \%$ para o cálcio e de $83 \%$ a $97 \%$ para o fósforo, concluindo que $60 \%$ a $70 \%$ da incorporação intra-uterina pode ser adquirida com as soluções parenterais habituais. Fator importante para haver estabilidade da solução e maior retenção é a relação Ca:P. Em termos de pesos desses elementos, as relações de $1,3: 1,0$ a 1,7:1,0 têm sido consideradas como resultando em retenção adequada de Ca e de $\mathrm{P}^{4}$. Rigo et al. $^{2}$, em 2000, consideraram que uma relação $C a: P$ de $1,7: 1,0$ permite o máximo de ministração e de retenção de cálcio e fósforo por via parenteral.

\section{Suprimento de cálcio e fósforo por nutrição enteral}

Quando ministramos Ca e P, quer sob a forma de leite materno com suplementos ou sob a forma de fórmulas especiais para prematuros, devemos considerar que os minerais retidos pelo organismo serão a resultante do total ministrado, do total absorvido pelo intestino e da quantidade eliminada pelos rins e fezes.

A absorção intestinal do cálcio ocorre por transporte ativo e por difusão facilitada. A absorção ativa localiza-se na parte proximal do intestino delgado, sendo dependente de vitamina D. A média de absorção de Ca em prematuros alimentados com fórmulas para pré-termo varia de $36 \%$ a $75 \%$ do ingerido, semelhante ao observado para o leite humano fortificado com cálcio ${ }^{4}$.

Quanto ao fósforo, a absorção no delgado ocorre por difusão simples, difusão facilitada e por co-transporte sódio-fosfato ${ }^{4}$. Também na absorção do fósforo há interferência da 1,25-dihidroxivitamina $D$ e do hormônio paratireoidiano. Observa-se que a retenção de fósforo é elevada com o leite humano sem suplementos, por ocorrer baixa concentração de fósforo, chegando a $90 \%$ do ingerido, sendo de aproximadamente $50 \%$ quando se tratarem de leites com teores elevados de fósforo 4 .

Vários fatores interferem na absorção do cálcio além da vitamina D. Em prematuros, os requerimentos de vitamina D são influenciados pelo armazenamento ao nascimento, que depende não somente dos estoques maternos, que variam em função da duração da gestação e da região geográfica². O cálcio, para ser absorvido, deve estar sob a forma iônica, pois frações insolúveis são pouco absorvidas. Apresenta melhor solubilidade quando se encontra sob a forma de cloreto, citrato e carbonato, comparado ao cálcio sob a forma de fosfato, devendo, portanto, ser evitado em fórmulas ${ }^{2}$. Também apresenta melhor solubilidade sob a forma de gluconato-glicerofosfato ${ }^{2}$. Observou-se que a quantidade e a qualidade da gordura influenciam na absorção do cálcio, formando sabões e reduzindo a absorção. No leite materno, a posição do palmitato no carbono 2 do triglicerídeo melhora a biodisponibilidade do cálcio.

\section{Conteúdo de cálcio do leite humano e de fórmulas}

Estudos reunindo dados de pesquisas sobre leite humano relatam que o conteúdo de cálcio no colostro inicial é, em média, de $160 \mathrm{mg} / \mathrm{l}$, passando no terceiro dia para $256 \mathrm{mg} / \mathrm{l}$ e persistindo nestes valores até 3 meses, quando declina lentamente até $176 \mathrm{mg} / \mathrm{l}$ ao redor de 1 ano ${ }^{5}$. Também o conteúdo do leite de mãe de pré-termo não difere do leite da mãe de termo em relação ao cálcio e ao fósforo no primeiro mês de vida.

Estes dados indicam que o conteúdo mineral do leite humano, no volume possível de ser ministrado, é insuficiente para manter a mineralização pós-natal de prematuros de muito baixo peso em níveis observados na fase intrauterina. Para tanto, desenvolveram-se suplementos nutricionais para o leite humano, com formulações variadas quanto ao conteúdo de cálcio e fósforo, proteínas e minerais, bem como fórmulas para pré-termo que se propõem a ministrar quantidades elevadas de cálcio e fósforo, no sentido de melhorar a mineralização óssea desses pequenos prematuros.

Observa-se diversificação na composição desses suplementos, na constituição das fórmulas, bem como na retenção de cálcio e fósforo, que, em última análise, é o fator fundamental da mineralização óssea. Assim, Koo \& Tsang ${ }^{4}$, em 1993, em pesquisas relatadas por seis autores, avaliaram o balanço mineral em prematuros com leite humano associado a cálcio e fósforo ou a suplementos comerciais e com fórmulas lácteas para pré-termo, com conteúdos variados de cálcio e fósforo, relatadas por sete autores. Também Rigo et al. ${ }^{2}$, em 2000, avaliando três diferentes suplementações para leite humano e 12 fórmulas para prétermo, mostraram grande variação na composição, absorção e retenção do cálcio e do fósforo. Apresentamos na Tabela 1 alguns dados retirados desses dois autores.

Observa-se grande variação no conteúdo de cálcio ministrado por kg/peso/dia, tanto para o leite humano suplementado como para as fórmulas. No leite humano, um fator importante para regular a retenção do cálcio é a suplementação do fósforo, mesmo porque o conteúdo de fósforo do leite humano é baixo. Nas fórmulas, a precipitação ou a menor solubilidade do cálcio podem interferir na sua absorção ${ }^{12}$. Entretanto, observa-se na Tabela 1 que algumas fórmulas com elevado conteúdo de cálcio resultam em retenção de cálcio compatível com as necessidades para uma adequada mineralização. Também nas fórmulas para pré-termo, uma relação $\mathrm{Ca}: \mathrm{P}$ adequada deve ser observada, propondo-se relação próxima a $2: 1$, avaliando-se a biodisponibilidade do sal de cálcio e a quantidade de fósforo empregada ${ }^{2}$.

Não há dúvidas de que a suplementação do leite humano com Ca e P leva ao aumento da quantidade de cálcio retido pelo prematuro. Entretanto, a análise do efeito sobre o osso não tem mostrado resultados muito evidentes. A avaliação pelo Cochrane Review, em $2004^{13}$, não conseguiu reunir 
Tabela 1 - Balanço mineral em RN de pré-termo recebendo nutrição enteral

\begin{tabular}{|c|c|c|c|c|c|c|c|}
\hline \multicolumn{8}{|c|}{ Leite materno e suplementos } \\
\hline & \multirow[t]{2}{*}{ Tipo de suplemento } & \multicolumn{3}{|c|}{ Cálcio (mg/kg/dia) } & \multicolumn{3}{|c|}{ Fósforo (mg/kg/dia) } \\
\hline & & Ingestão & Absorção & Retenção & Ingestão & Absorção & Retenção \\
\hline Salle et al. ${ }^{6}$ & $\mathrm{Ca}+\mathrm{P}$ & 90 & 66 & 63 & 62 & 58 & 53 \\
\hline Ehrenkranz et al. ${ }^{7}$ & $\begin{array}{l}\text { Suplemento baseado } \\
\text { em leite de vaca }\end{array}$ & 123 & 92 & 82 & 72 & 61 & 58 \\
\hline Schanler et al. ${ }^{8}$ & $\begin{array}{l}\text { Suplemento baseado } \\
\text { em leite de vaca }\end{array}$ & 137 & 48 & 43 & 71 & 63 & 39 \\
\hline \multicolumn{8}{|c|}{ Fórmulas para pré-termo } \\
\hline Rigo et al. ${ }^{9}$ & PT $F_{1}$ & $91 \pm 14$ & $51 \pm 17$ & $45 \pm 18$ & $63 \pm 9$ & $89 \pm 6$ & $42 \pm 8$ \\
\hline Rigo et al. ${ }^{10}$ & PT $F_{6}$ & $135 \pm 4$ & $46 \pm 13$ & $59 \pm 17$ & $88 \pm 3$ & $91 \pm 5$ & $53 \pm 7$ \\
\hline Schanler et al. ${ }^{8}$ & & 147 & 79 & 74 & 69 & 61 & 50 \\
\hline Rowe et al. ${ }^{11}$ & & 219 & 166 & 159 & 116 & 89 & 87 \\
\hline
\end{tabular}

Adaptado de Koo \& Tsang $^{4}$ e Rigo et $\mathrm{al}^{2}$.

pesquisas randomizadas em neonatos suplementados apenas com cálcio e fósforo e que avaliassem o crescimento e o metabolismo ósseo de prematuros, pois a maioria das pesquisas avalia apenas parâmetros metabólicos e, principalmente, o uso de suplementos de leite humano com múltiplos componentes.

Quanto à análise de várias pesquisas pelo Cochrane Review ${ }^{14}$ com leite humano com suplementos e componentes variados, como proteínas, gordura, carboidrato, minerais, eletrólitos e microelementos, com pelo menos dois componentes entre os citados e iniciada quando o recémnascido tolerasse uma ingestão pré-definida, constatou-se que ocorre, a curto prazo, aumento do ganho de peso, do comprimento e do perímetro cefálico, sem diferenças nos níveis de fosfatase alcalina do soro. Quanto à mineralização óssea, os resultados são conflitantes, com duas pesquisas referindo somente aumento do CMO do rádio ${ }^{15}$ e aumento no CMO total ${ }^{16}$, e dois autores citados ${ }^{17,18}$ não mostrando diferenças no CMO total com a suplementação. Não foram observadas fraturas, hipercalcemia ou outras manifestações clínicas.

As fórmulas de pré-termo, quando comparadas ao leite humano com suplemento, produzem maior crescimento longitudinal; entretanto, a incorporação mineral é semeIhante, apesar de menor do que a observada intra-útero ${ }^{2}$.

$\mathrm{Na}$ decisão sobre qual tipo de alimentação escolher, outros fatores favoráveis ao uso do leite materno com suplementos devem ser levados em conta. Estudos com balanço metabólico indicam que a absorção do cálcio é uma função linear da ingestão, na faixa de ingestão variando de 40 a $120 \mathrm{mg} / \mathrm{kg} / \mathrm{dia}$ com leite humano suplementado ou com fórmulas para pré-termo ${ }^{5}$.

\section{Doença óssea metabólica}

Compreende distúrbios de mineralização óssea encontrados em prematuros, variando desde um estado de hipomineralização, definido como osteopenia da prematuridade, até alterações mais intensas, caracterizando um quadro de raquitismo da prematuridade. A freqüência é maior em prematuros com peso abaixo de $1.000 \mathrm{~g}$, nos quais é observada em $50 \%$, e naqueles com peso inferior a $1.500 \mathrm{~g}$, ocorrendo em $30 \%{ }^{2}$.

O diagnóstico clínico ocorre entre 2 e 4 meses e caracteriza-se por sinais de raquitismo, com achatamento posterior do crânio, espessamento das junções condrocostais, alargamento das porções distais do rádio e do cúbito, aumento da fontanela anterior e das bossas frontais. As fraturas de costelas e ossos longos incidem em $10 \%$ dos prematuros de muito baixo peso. Acompanhando o quadro clínico, pode haver redução no crescimento. Este quadro caracteriza-se como raquitismo da prematuridade, entretanto, o quadro mais freqüente é o de hipomineralização, que pode ser evidenciado ao raio $X$ de ossos longos. Nesta fase, quando aparecem sinais radiológicos, já ocorreu redução da densidade óssea com perda de $40 \%$ dos minerais do osso.

O diagnóstico radiológico é impreciso e depende da gravidade e da duração da deficiência de minerais. Avaliação mais precisa é efetuada ao determinar-se o CMO por densitometria óssea com o aparelho de absorção de fótons simples ou duplo, quando são empregados dois feixes de energia. O método duplo é mais fidedigno para medir o CMO do corpo todo.

Quanto ao quadro bioquímico, o cálcio sérico pode apresentar níveis normais ou baixos, o fósforo sérico é 
baixo, e a atividade da fosfatase alcalina se eleva assim como a osteocalcina. Indicadores bioquímicos, como a fosfatase alcalina, têm sido considerados para identificar prematuros com doença óssea metabólica. O aumento na fosfatase alcalina, com ponto de corte correspondente a cinco vezes o valor de referência para adultos normais, tem sido empregado na prática clínica. Entretanto, observam-se conclusões conflitantes em pesquisas relacionando a fosfatase alcalina com o CMO determinado por absorção por fótons. Assim, alguns autores relatam associação fraca entre a fosfatase e o fósforo sérico com o $\mathrm{CMO}$, enquanto outros autores, como Faerk et al. ${ }^{19}$, em 2002, não observaram associação entre o CMO e a fosfatase alcalina ou o fósforo sérico em prematuros, por ocasião da idade correspondente ao termo. Observaram, entretanto, associação negativa significativa entre a fosfatase e o fósforo sérico quando avaliaram 127 prematuros com idades inferiores a 32 semanas de gestação. Empregaram a absorção por dupla energia de raio $\mathrm{X}$ e scanner de corpo inteiro, método mais preciso do que os empregados por outros pesquisadores, e concluíram que medidas de rotina de fosfatase alcalina e fósforo sérico não são úteis para predizer a evolução da mineralização óssea em prematuros.

Embora se considere que a deficiência em cálcio e fósforo seja a causa fundamental da doença óssea do prematuro, há indicações de que o aumento na reabsorção óssea ${ }^{20}$ contribui para a doença óssea do prematuro, desde que o aparecimento de marcadores de reabsorção, como a hidroxiprolina proveniente do turnover do colágeno, foi identificado em sangue de prematuros. Além desse aspecto, mesmo com fórmulas com altas concentrações de cálcio e fósforo, o CMO de crianças prematuras por ocasião da alta é inferior aos valores de referência. Segundo Miller ${ }^{20}$, em 2003, as variáveis que explicariam as diferenças na reabsorção óssea do pré-termo comparado ao termo seriam fatores biomecânicos, pelos quais forças atuando sobre os ossos estimulariam osteoblastos que produzem osso e osteclastos que reabsorvem o osso, resultando em um processo de remodelação óssea. Essas forças resultariam de movimentos efetuados pelos fetos contra a parede uterina, ao contrário do prematuro em ambiente extrauterino. Miller ${ }^{20}$, em 2003, sugere que se reavalie a quantidade de minerais a serem ofertados no período pós-natal, pois estes podem não corresponder às necessidades intrauterinas.

Outros fatores observados em prematuros extremos que influenciam o crescimento e a mineralização óssea são a prolongada nutrição por via parenteral e o uso de drogas com efeito hipercalciúrico, como a furosemida e as metilxantinas.

\section{Efeitos da nutrição após o nascimento sobre o crescimento e a mineralização óssea a curto e longo prazo}

O prematuro de extremo baixo peso, por ocasião da alta hospitalar, freqüentemente apresenta restrição do crescimento ${ }^{21}$ e hipomineralização óssea, decorrentes das dificuldades em receber nutrição adequada. Estudos epidemiológicos sugerem que a restrição do crescimento intra-uterino e pós-natal pode levar a doenças no adulto. Entretanto, as conseqüências tardias sobre o esqueleto ainda necessitam ser determinadas.

Efeitos a curto prazo de deficiente ingestão de cálcio e fósforo, levando a CMO reduzido por ocasião da alta, têm sido relatados por diversos autores. Segundo Rigo et al. ${ }^{2}$, em 2000, a recuperação da mineralização pode ocorrer ao redor da $12^{a}$ semana pós-termo. Entretanto, estudos a longo prazo são escassos e difíceis de serem avaliados em função dos diferentes protocolos de alimentação de prematuros. Há indícios de que o déficit no CMO desapareça na infância, principalmente se corrigido em função de dados antropométricos. Sessenta e quatro prematuros com idades gestacionais inferiores a 32 semanas e, entre estes, 30 prematuros extremos (idade gestacional inferior a 28 semanas) receberam durante a internação leite materno suplementado. Após a alta, foram amamentados com leite materno ou com fórmula padrão. Quando atingiram entre 5,0 e $7,0 \mathrm{~kg}$, o CMO da coluna lombar foi avaliado por dupla absorção. Por ocasião da alta, $70 \%$ deles havia desenvolvido déficit do crescimento. Com o leite materno exclusivo, foi mantido o ganho de peso linear, porém, com risco aumentado (OR 7,0; IC95\% 1,2-41,7) de apresentar valores baixos de CMO. Como fator de risco para a hipomineralização, foi indicado o fósforo sérico baixo às 6 semanas pósnatal $^{22}$. Concluem os autores que deve ser avaliada a possibilidade de fortificação do leite humano após a alta hospitalar ${ }^{22}$. Em outra pesquisa ${ }^{23}$, dois grupos de prematuros que receberam leite humano sem e com suplementação de cálcio ( $108 \mathrm{mg} / \mathrm{kg} / \mathrm{dia}$ ) e de fósforo ( $53 \mathrm{mg} / \mathrm{kg} / \mathrm{dia})$, até atingir $2.000 \mathrm{~g}$, foram examinados aos 3 meses de idade. 0 CMO foi $36 \%$ maior no grupo suplementado. Entretanto, quando examinados aos 9 e 11 anos de idade, o estado de mineralização foi igual em ambos os grupos e adequado para o tamanho 23 .

Fewtrell et al. ${ }^{24}$, em 1999, ao avaliarem crianças aos 8-12 anos que haviam nascido prematuras e sido alimentadas com leite humano, fórmula para pré-termo ou fórmula de termo, concluíram que os prematuros permaneceram menores do que os recém-nascidos de termo aos 8-12 anos e que o CMO, embora menor, estava adequado ao tamanho, concluindo que as diferenças na ingestão de minerais não afetaram a massa óssea de crianças nascidas pré-termo e examinadas aos 8-12 anos. Consideram que, para melhorar a massa óssea, há necessidade de melhorar o crescimento.

A maior parte das pesquisas sobre conseqüências de hipomineralização em etapas precoces da vida refere-se à avaliação a longo prazo de coortes analisadas em anos anteriores e que, conseqüentemente, apresentam falhas, já que não foram desenhadas com esta finalidade. Em geral, a casuística é pequena, e por vezes comparam prematuros que receberam, à época, dietas insuficientes do ponto de vista de nutrição mineral. Também a casuística não está voltada para prematuros de muito baixo peso ou de extremo baixo peso e, portanto, mais suscetíveis à osteopenia, além de não haver referências à 
nutrição pós-alta. Portanto, pesquisas randomizadas e controladas com prematuros extremos poderão indicar as conseqüências tardias da hipomineralização no período pós-natal.

\section{Nutrição do prematuro após a alta e efeitos sobre a mineralização óssea}

Prematuros de extremo baixo peso apresentam com freqüência, por ocasião da alta hospitalar, importante restrição de crescimento e complicações que podem, após a alta, afetar a ingestão de nutrientes e acarretar déficit protéico e energético, além de deficiente mineralização. Apesar da maioria destes prematuros apresentar catch up growth após a alta hospitalar, o crescimento ainda é menor quando comparado ao de recém-nascidos a termo.

Atualmente, há dados sugerindo que fórmulas enriquecidas com nutrientes para prematuros, empregadas após a alta hospitalar, podem aumentar significativamente o peso, o comprimento, o perímetro cefálico e a mineralização óssea, comparadas a fórmulas-padrão para nascidos a termo ${ }^{25,26}$. Os efeitos sobre o crescimento são mais evidentes nos primeiros meses e em recém-nascidos do sexo masculino 26 .

Dois tipos de fórmulas enriquecidas têm sido utilizados: fórmulas especialmente preparadas, denominadas de fórmulas pós-alta, e fórmulas para pré-termo semelhantes às usadas durante a internação. Também são empregadas como suplementos para leite materno. As fórmulas pós-alta caracterizam-se por apresentar maior conteúdo protéico, energético, suplementos de $\mathrm{Ca}, \mathrm{P}$, zinco e microelementos $^{27}$. Os trabalhos iniciais de Lucas et al. 28,29 , alimentando após a alta hospitalar prematuros com peso inferior a 1.850 $\mathrm{g}$, com fórmula enriquecida com proteínas e minerais, mostraram maior crescimento e mineralização do que o observado com fórmulas para recém-nascidos a termo, criando, assim, o conceito de fórmula pós-alta. Griffin ${ }^{30}$, em 2002, faz uma revisão das diversas formas de suplementação alimentar de prematuros após a alta. Os benefícios são pouco evidentes e por vezes controversos, havendo necessidade de novas avaliações que resultem em conclusões mais concretas. Há referência a pesquisa em que prematuros, recebendo fórmula pós-alta até 9 meses de idade pósnatal, apresentaram maior CMO do que recém-nascidos a termo alimentados com fórmulas. Também estes apresentaram maior peso, comprimento e perímetro cefálico. Outros autores, usando fórmulas semelhantes e pelo mesmo período de tempo, relataram aumento de peso e comprimento aos 9 meses, porém, aos 18 meses, persistiram diferenças apenas no comprimento. Em outra pesquisa, em que fórmula pós-alta foi ministrada até 12 meses de idade corrigida, os prematuros passaram a ingerir menor volume, igualando as quantidades de energia ao correspondente à fórmula de termo. A diferença no peso ocorreu em etapas precoces.

Quanto a continuar após a alta hospitalar com as fórmulas usadas para recém-nascidos pré-termo, há poucas pesquisas. Entretanto, estas fórmulas, variadas em sua composição, costumam apresentar quantidades elevadas de vitaminas e minerais, calculadas para permitir crescimento e acréscimo mineral semelhantes ao intra-uterino e que não necessariamente correspondem às necessidades pós-natais. Observou-se aumento na concentração de zinco plasmático e melhora do comprimento e perímetro cefálico apenas em meninos. Outra pesquisa não mostrou diferenças no cálcio, fósforo e fosfatase alcalina do soro.

Fewtrell27, em artigo de revisão, considera que as fórmulas pós-alta ou de pré-termo resultam em melhora do crescimento, sendo preferíveis às fórmulas de termo após a alta de prematuros.

As pesquisas sobre suplementação do leite materno no período pós-alta referem-se a suplementos pontuais, com aumento de proteínas, vitaminas ou minerais, ou, ainda, à associação desses componentes. A suplementação de leite materno com cálcio, fósforo e vitamina $D$, após a alta hospitalar, reduziu a incidência de hipofosfatemia. Fórmulas acrescentadas com cálcio e fósforo e empregadas por curto tempo após a alta hospitalar, durante 8 semanas, mostraram aumento do CMO após este período, podendo não persistir quando analisadas em período mais longo ${ }^{30}$.

\section{Outros minerais: elementos traço ou microelementos}

Microelementos, oligoelementos ou elementos traço com importância em nutrição humana são o zinco, o cobre, o selênio, o cromo, o molibdênio, o manganês, o iodo e o ferro. Embora quantitativamente representem pequena fração do total do conteúdo mineral do corpo humano, apresentam papel importante em várias vias metabólicas. Os prematuros podem apresentar deficiências, mesmo que não traduzidas por quadro clínico, em decorrência de estoques baixos ao nascimento, uma vez que a incorporação desses minerais ocorre no último trimestre da gestação. Muito pouco se sabe sobre o metabolismo desses elementos traço na nutrição de prematuros extremos, pois os conhecimentos referem-se a prematuros ou prematuros de muito baixo peso.

\section{Selênio}

Sua importância vem crescendo em todas as faixas etárias, pois é participante de selenoenzimas com distintas funções, incluindo a glutationa peroxidase, que se destaca como enzima antioxidante nas membranas celulares. Estas enzimas impedem a formação de radicais livres, reduzindo os peróxidos lipídeos e o peróxido de hidrogênio e protegendo o organismo da agressão oxidativa. Outra função do selênio é seu papel na imunocompetência. Neutrófilos e macrófagos de animais deficientes em selênio têm baixa concentração de glutationa peroxidase, o que pode afetar suas propriedades antimicrobianas ${ }^{5,31}$.

O prematuro de extremo baixo peso encontra-se em situação de risco para condições que tenham em sua fisiopatologia a agressão por radicais livres, como observado na displasia broncopulmonar, na retinopatia da prematuridade, na hemorragia peri-intraventricular e na enterocolite necrosante. Também pela freqüência com 
que recebem oxigênio, são suscetíveis a estresse oxidativo e produção de oxigênio reativo. Embora haja indícios da participação da deficiência de selênio em doenças de prematuros, ainda faltam dados para comprovar a real importância. Em artigo de revisão, Klein ${ }^{5}$ relata pesquisas relacionando a deficiência de selênio a doenças de prematuros. Assim, oito prematuros que desenvolveram Síndrome do Desconforto Respiratório e que não receberam selênio oral ou parenteral apresentaram queda rápida dos níveis sangüíneos 2 semanas após o nascimento. Também prematuros com doença pulmonar crônica e que não receberam suplementação de selênio apresentaram queda acentuada nas concentrações de selênio. Na Nova Zelândia, país em que o solo e os alimentos são pobres em selênio, níveis baixos de selênio em 79 prematuros de muito baixo peso associaram-se ao aumento da morbidade, e as concentrações plasmáticas foram significativamente mais baixas em crianças dependentes de oxigênio aos 28 dias pós-natal. As conseqüências de concentrações baixas de selênio em recém-nascidos não estão definidas. Em prematuros, têm sido sugeridas como fator de risco para doença pulmonar crônica e retinopatia da prematuridade, havendo incerteza se a suplementação de selênio reduz o risco para essas doenças.

\section{Selênio no feto e no recém-nascido}

Os mecanismos de controle da passagem materno-fetal de selênio ainda não estão esclarecidos. O transporte através da placenta é limitado, de modo que a concentração no feto é relativamente baixa, correspondendo no sangue do cordão a aproximadamente $65 \%$ do nível sérico materno. Observamos em prematuros de muito baixo peso concentrações de selênio do cordão sem diferenças significativas quando comparados a recém-nascidos a termo, quer adequados ou pequenos para a idade gestacional e correspondendo a aproximadamente $80 \%$ dos valores maternos 32 . Entretanto, Makhoul et al. ${ }^{33}$, avaliando selênio em sangue de cordão de recém-nascidos desde 24 até 42 semanas de idade gestacional, mostraram associação significativa com a idade gestacional e aumento significativo da concentração após 36 semanas.

Não há dados sobre o conteúdo fetal de selênio, estimando-se que a incorporação corresponda a $1 \mu \mathrm{g} / \mathrm{kg} / \mathrm{dia}$. 0 selênio é estocado no fígado fetal entre 20 e 40 semanas, podendo ser responsável pelos baixos níveis observados em recém-nascidos pré-termo. Na literatura, são relatadas pesquisas que analisam as concentrações de selênio em prematuros de muito baixo peso, mostrando que as concentrações plasmáticas de selênio e da glutationa peroxidase correlacionam-se com o peso de nascimento e que a atividade da enzima glutationa peroxidase corresponde, nos prematuros de muito baixo peso, a um terço do observado em recém-nascidos de termo ${ }^{34}$.

Em prematuros com problemas respiratórios, em nutrição parenteral sem suplementação de selênio nos primeiros 14 dias, foi observado declínio importante do selênio nos primeiros dias de vida. As concentrações se elevaram quando foi iniciada uma fórmula com selênio, exceto para um recém-nascido que desenvolveu displasia broncopulmonar ${ }^{35}$. Outros autores têm mostrado queda progressiva da atividade da glutationa peroxidase e do selênio dos eritrócitos de recém-nascidos prematuros a partir da segunda semana de nascimento, quando alimentados com fórmula láctea padronizada.

Em pesquisa realizada na Unidade Neonatal da Faculdade de Medicina de Botucatu da UNESP, avaliamos as concentrações de selênio sérico em três classes de recémnascidos: pré-termo de muito baixo peso, termo adequado e termo pequeno para a idade gestacional, desde o nascimento até 30 dias de idade cronológica. Estes recémnascidos não receberam suplementação de selênio em nutrição parenteral ou enteral. Os níveis de selênio do cordão foram inferiores aos níveis maternos. As únicas diferenças observadas foram níveis significativamente menores nos pré-termo de muito baixo peso no $3^{\circ}, 15^{\circ}$ e $30^{\circ}$ dias em relação aos neonatos de termo adequados e aos de termo pequenos para a idade gestacional, bem como uma queda progressiva do selênio no primeiro mês de vida, somente em prematuros ${ }^{32}$. Estes resultados nos permitem concluir sobre a necessidade de suplementar a nutrição do prematuro de muito baixo peso com selênio na nutrição parenteral e enteral. Esta conduta é referendada por Darlow et al. ${ }^{36}$, em estudo multicêntrico, randomizado e duplocego em 534 prematuros de muito baixo peso, avaliados aos 28 dias e 36 semanas de idade pós-concepcional. O grupo tratado recebeu $7 \mu \mathrm{g} / \mathrm{kg} / \mathrm{dia}$ quando em parenteral e $5 \mu \mathrm{g} / \mathrm{kg} /$ dia acrescido ao leite materno ou fórmula. Os autores observaram que os valores da glutationa peroxidase e do selênio do plasma foram significativamente mais baixos no grupo não suplementado aos 28 dias e 36 semanas de idade corrigida. Estas observações também foram confirmadas pela maioria das pesquisas sobre selênio em prematuros. Entretanto, resta avaliar se a suplementação traz benefícios na prevenção de doenças dos recémnascidos. Em metanálise efetuada por Darlow \& Austin 37 , três pesquisas preencheram os requisitos e reuniram 297 recém-nascidos com suplementação de selênio e 290 controles. Dois estudos incluíam prematuros extremos. Dentre as complicações, avaliaram a necessidade de oxigênio aos 28 dias, episódios de sepse, morte durante internação, doença pulmonar crônica (necessidade de oxigênio aos 28 dias e com 36 semanas de idade pósconceptual) e retinopatia. Como conclusão, relatam que a suplementação de selênio reduziu um ou mais episódios de sepse tardia, não havendo significância para as demais doenças ou complicações avaliadas.

As quantidades de selênio empregadas para a suplementação para prematuros são variadas, sendo recomendado nos Estados Unidos $2 \mu \mathrm{g} / \mathrm{kg} /$ dia em nutrição parenteral. Entretanto, na metanálise, são sugeridos $3 \mu \mathrm{g} / \mathrm{kg} / \mathrm{dia}$ para manter as concentrações nos níveis do cordão 37 . Para elevar acima dos níveis do cordão e próximo aos valores de recém-nascidos de termo amamentados, recomendam 5-7 $\mu \mathrm{g} / \mathrm{kg} / \mathrm{dia}$. Outros fatores que influenciam os níveis de selênio são as vias utilizadas, as formas bioquímicas e o conteúdo de selênio das fórmulas empregadas $^{37}$. 
Especialistas reunidos em painel programado pela Food and Drug Administration e American Society for Nutritional Sciences ${ }^{5}$ recomendam uma concentração mínima de selênio de $1,8 \mu \mathrm{g} / 100 \mathrm{kcal}$ e máxima de $5,0 \mu \mathrm{g} / 100 \mathrm{kcal}$ nas fórmulas para pré-termo.

\section{Zinco}

O zinco talvez seja o microelemento mais estudado em nutrição do recém-nascido, pois é importante para o crescimento, diferenciação celular, metabolismo de proteínas, carboidratos e lipídeos. Tem papel na estrutura hormonal e em fatores de transcrição genética ${ }^{38}$. Sinais de deficiência subclínica de zinco podem ocorrer e assemelham-se à deficiência de outros componentes da dieta em virtude de seu vasto leque de atuação, caso o prematuro permaneça por tempo prolongado em nutrição parenteral sem adição de zinco. Manifestações mais características surgem após 3 meses de idade, incluindo perda de peso, dificuldade em crescer, dermatite ao redor de orifícios, glossite e suscetibilidade aumentada às infecções ${ }^{31}$. Alguns fatores contribuem para o aparecimento de deficiências, como o nascimento prematuro, privando o recém-nascido da incorporação fetal do zinco, que, à semelhança dos demais micronutrientes, acumula-se no terceiro trimestre de gestação. Também apresentam trato gastrointestinal imaturo, resultando em balanço negativo de zinco, com excreção de zinco pelo trato intestinal. Estudos com isótopos marcados mostram que o prematuro tem capacidade de captar zinco exógeno, aumentando sua absorção e reduzindo a excreção, mas não se conhece a evolução deste comportamento em prematuros extremos. Prematuros podem absorver de $25 \%$ a $40 \%$ do zinco da dieta 5 . Além da menor absorção por via enteral e da menor reserva hepática, quando comparado a outros microelementos, os componentes de fórmulas, como o ferro, podem afetar a biodisponibilidade do zinco quando houver alta relação Fe:Zn. A interferência do ferro sobre o zinco é observada em adultos e é discutível em prematuros. Entretanto, Klein ${ }^{5}$ refere que, nas fórmulas, deve ser utilizada uma relação $\mathrm{Fe}: Z n$ de 2:1, com o máximo de 3,0 $\mathrm{mg}$ de zinco para $1,5 \mathrm{mg}$ de ferro para $100 \mathrm{kcal}$.

A biodisponibilidade do zinco do leite materno é maior do que a do leite de vaca, portanto, nas fórmulas, a concentração de zinco a ser ministrada deve ser elevada. A diferença na biodisponibilidade deve-se à forte ligação do zinco com a caseína ${ }^{5}$. Demonstrou-se que $60 \%$ do zinco do leite humano de pré-termo é absorvido, em comparação com $36 \%$ do zinco do leite humano suplementado e com $14 \%$ em fórmula para prematuros. A concentração do zinco no leite humano cai rapidamente a partir do colostro, quando os níveis são os mais elevados ${ }^{38}$.

O crescimento é o fator principal para determinar as necessidades de zinco em prematuros. Cálculos das necessidades para prematuros entre 24 e 28 semanas de idade gestacional indicam requerimento de $600 \mu \mathrm{g} / \mathrm{dia}$ de zinco para formação de tecido novo (excluindo-se zinco para armazenamento), para proporcionar crescimento comparável a um feto de 40 semanas de idade pósconceptual. A liberação de zinco hepático de um recém- nascido de $1.000 \mathrm{~g}$ é, em média, de $150 \mu \mathrm{g} / \mathrm{dia}$. Prematuros extremos têm menores reservas, e a depleção ocorre mais cedo, ao redor de 32-36 semanas. O cálculo do zinco da dieta, para que haja crescimento ótimo, será de $500 \mu \mathrm{g} / \mathrm{kg} /$ dia para recém-nascido com aproximadamente $1.000 \mathrm{~g}$ de peso e 27 semanas de idade gestacional; $400 \mu \mathrm{g} / \mathrm{kg} /$ dia para recém-nascido entre 1.500 $2.000 \mathrm{~g}$ (30-32 semanas); e 200-300 $\mathrm{\mu g} / \mathrm{kg} / \mathrm{dia}$ para recém-nascido entre $2.500-3.500 \mathrm{~g}$ (35-40 semanas) ${ }^{5}$.

Considerando a baixa absorção do zinco ingerido em fórmulas, o painel de especialistas em $2002^{5}$ recomendou que, nas fórmulas para pré-termo, as concentrações mínimas e máximas sejam 1,1 mg/100 kcal e 1,5 mg/100 kcal. No período inicial, autores recomendam $500-800 \mu \mathrm{g} / \mathrm{kg} / \mathrm{dia}$, aumentando para $1 \mathrm{mg} / \mathrm{kg} /$ dia quando o crescimento começar a estabelecer-se ${ }^{38}$. Por via parenteral, preconiza-se no prematuro estável $400 \mu \mathrm{g} / \mathrm{kg} /$ dia e, no período de transição (até 2 semanas), $150 \mu \mathrm{g} / \mathrm{kg} / \mathrm{dia}^{38}$.

\section{Outros minerais}

$\mathrm{Na}$ literatura, o enfoque sobre o ferro visa mais os aspectos relacionados com morbidade do prematuro do que seus aspectos nutricionais. As pesquisas estão voltadas para a prevenção e o tratamento da anemia da prematuridade.

Minerais como o cobre, o iodo, o manganês e o molibdênio têm sido pesquisados em nutrição de prematuro com vistas à adaptação de fórmulas especiais para pré-termo e soluções para a nutrição parenteral. Quase não há referências sobre os requerimentos de recémnascidos pré-termo extremo, e, portanto, temos que nos valer das publicações para recém-nascidos de termo ou pré-termo de muito baixo peso. Também não são descritas manifestações clínicas decorrentes de deficiência no período neonatal, motivo pelo qual suas características são menos referidas na prática diária.

Entre os minerais, chamamos a atenção para o cobre, elemento importante na constituição de enzimas como a superóxido dismutase, que protege as membranas celulares contra danos oxidativos. É armazenado no fígado fetal, ligado à metalotioneína em quantidades superiores às observadas no fígado de adultos. Entretanto, o transporte do fígado aos tecidos é feito pela ceruloplasmina, cujos níveis são baixos no recém-nascido, iniciando-se sua produção 6 a 12 semanas após o nascimento5,31,38. A deficiência é rara no período neonatal, e, quando presente, ocorre anemia hipocrômica resistente à terapêutica com ferro, neutropenia, osteoporose, manifestações cutâneas e dificuldade em ganhar peso. Há poucos dados sobre requerimentos de cobre em prematuros. Quando alimentados com leite materno, recebem quantidades suficientes. Em parenteral por tempo prolongado, preconiza-se empregar $20 \mu \mathrm{g} / \mathrm{kg} /$ dia e não ministrar quando houver colestase, pois o mesmo é excretado pela bile ${ }^{38}$.

Esperamos que mais pesquisas sejam efetuadas sobre minerais na nutrição de prematuros, para que as formulações empregadas em nutrição possam ser mais equilibradas 
quanto à composição dos minerais e mais adequadas às necessidades dos prematuros extremos, que são os que permanecem por maior tempo em nutrição parenteral.

\section{Referências}

1. Greer FR, Tsang RG. Calcium, phosphorus, magnesium and vitamin $D$ requirements for the preterm infant. In: Tsang RC, editor. Vitamin and minerals requirements in preterm infants. New York: Marcel Deker; 1985. p. 99-136.

2. Rigo J, De Curtis M, Pieltain C, Picaud J, Salle BL, Santerre J. Bone mineral metabolism in the micropremie. Clin Perinatol. $2000 ; 27: 147-70$.

3. Venkataraman PS, Tsang RC, Chen IW, Sperling MA. Pathogenesis of early neonatal hypocalcaemia: studies of serum calcitonin, gastrin, and plasma glucagon. J Pediatr. 1987;110:559-603.

4. Koo WW, Tsang RC. Calcium, magnesium, phosphorus and vitamin D. In: Tsang RC, Lucas A, Uauy R, Zlotkin S, editors. Nutrition needs of the preterm infant. Scientific basis and practical guidelines. Baltimore: Williams \& Wilkins; 1993. p. 135-55.

5. Klein $\mathrm{C}$. Nutrient requirements for preterm-infant formulas: 10. Minerals: calcium and phosphorus. J Nutr. 2002;132(6 Suppl 1):S1395-577.

6. Salle B, Senterre J, Putet G, Rigo J. Effects of calcium and phosphorus supplementation on calcium retention and fat absorption in preterm infants fed pooled human milk. J Pediatr Gastroenterol Nutr. 1986;5:638-42.

7. Ehrenkranz RA, Gettner PA, Nelli CM. Nutrient balance studies in premature infants fed premature formula or fortified human milk. J Pediatr Gastroenterol Nutr. 1989;8:58-67.

8. Schanler RJ, Abrams AS, Garza C. Bioavailability of calcium and phosphorus in human milk fortifiers and formula for very low birth weight infants. J Pediatr. 1988;113:95-100.

9. Rigo J, Salle BL, Picaud JC, Putet G, Senterre J. Nutritional evaluation of protein hydrolysate formulas. Eur J Clin Nutr. 1995;49 Suppl 1:S26-38.

10. Rigo J, Picaud JC, Lapellone A, Salle LB, Senterre, J. Metabolic balance and plasma AA concentrations in VLBW infants fed a new acidic whey hydrolysate preterm formula. J Pediatr Gastroenterol Nutr. 1997;24:491A.

11. Rowe JC, Goez CA, Carey DE, Horak E. Achievement of in uterus retention of calcium and phosphorus accompanied by high calcium excretion in very low birth weight infants fed a fortified formula. J Pediatr. 1987;110:581-5.

12. Salle BL, Senterre J, Putet G. Calcium, phosphorus, magnesium and vitamin $D$ requirements in premature infants. In: Salle $B L$, Swyer PR, editors. Nutrition of the low birth weight infant. Nestlé Nutrition Workshop Series, vol. 32. New York: Raven Press; 1993. p. $125-34$

13. Kuschel CA, Harding JE. Calcium and phosphorus supplementation of human milk for preterm infants. Cochrane Database Syst Rev. 2001;(4):CD003310.

14. Kuschel CA, Harding JE. Multicomponent fortified human milk for promoting growth in preterm infants. Cochrane Database Syst Rev. 2004;(1):CD000343.

15. Mondanlou HD, Lim MO, Hansen GW, Sickles V. Growth, biochemical status and mineral metabolism in very-low birth weight infants receiving fortified preterm human milk. J Pediatr Gastroenterol Nutr. 1986;5:762-7.

16. Pettifor JM, Rajah R, Venter A, Moodley GP, Opperman L, Cavaleros $\mathrm{M}$, et al. Bone mineralization and mineral homeostasis in very-low-birth-weight infants fed either human milk or fortified human milk. J Pediatr Gastroenterol Nutr. 1989;8: 217-24.

17. Wauben IP, Atkinson SA, Grad TL, Shah JK, Paes B. Moderate nutrient supplementation of mother's milk for preterm infants supports adequate bone mass and short-term growth: a randomized, controlled trial. Am J Clin Nutr. 1998;67:465-72.

18. Faerk J, Petersen B, Petersen S, Michaelsen KF. Diet and bone mineral content at term in premature infants. Pediatr Res. 2000;47:148-56.
19. Faerk J, Petersen B, Petersen S, Michaelsen KF. Bone mineralisation in premature infants cannot be predicted from serum alkaline phosphatase or serum phosphate. Arch Dis Child Fetal Neonatal Ed. 2002;87:133-6.

20. Miller ME. The bone disease of preterm birth: a biomechanical perspective. Pediatr Res. 2003;53:10-5.

21. Berry MA, Conrad H, Usher RH. Growth of very premature infants fed hyperalimenation and calcium-supplemented formula. Pediatrics. 1997; 100:647-53.

22. Kurl S, Heinonen K, Lausimies E. Pre-and post-discharge feeding of very preterm infants: impact on growth and bone mineralization. Clin Physiol Func Imagin. 2003;23:182-9.

23. Backström MC, Mäki R, Kuusela AL, Sievänin $H$, Koivisto A-M, Koshinen $M$, et al. The long-term effect of early mineral, vitamin $D$, and breast milk intake on bone mineral status in 9-11-yearold children born prematurely. J Pediatr Gastroenterol Nutr. 1999;29:575-82.

24. Fewtrell MS, Prentice A, Jones SC, Bishop NJ, Stirling D, Buffenstein $\mathrm{R}$, et al. Bone mineralization and turnover in preterm infants 8-12 years of age: the effect of early diet. J Bone Miner Res. $1999 ; 14: 810-20$.

25. Carver JD, Wer PYK, Hall RT, Ziegler EE, Sosa R, Jacobs J, et al. Growth of preterm infants fed nutrient enriched or term formula after hospital discharge. Pediatrics. 2001;107:683-9.

26. Carver JD. Advances in nutritional modifications of infant formulas. Am J Clin Nutr. 2003;77:S1550-4.

27. Fewtrel MS. Growth and nutrition after discharge. Semin Neonatol. 2003;8:169-70.

28. Lucas A, King F, Bishop NB. Postdischarge formula consumption in infants born preterm. Arch Dis Child. 1992;67:691-2.

29. Lucas A, Fewtrell MS, Morley R, Singhal A, Abbot RA, Isaaco E, et al. Randomized trial of nutrient-enriched formula versus standard formula for postdischarge preterm infants. Pediatrics. 2001;108:703-11.

30. Griffin IJ. Postdischarge nutrition for high risk neonate. Clin Perinatol. 2002;29:327-44.

31. Agget PJ. Trace elements of the micropremie. Clin Perinatol. 2000;27:119-29.

32. Daher S, Trindade CE, Rezende C, Miranda A, Crossi R. Blood selenium levels of very low birth weight infants during the first month of life. Pediatr Res. 2001;49:297A.

33. Makhoul IR, Sammour RN, Diamond E, Shohat I, Tamir A, Shamir R. Selenium concentrations in maternal and umbilical cord blood at 24-42 weeks of gestation: basis for optimization of selenium supplementation to premature infants. Clin Nutr. 2004;23:373-81.

34. Locktich G, Jacobson B, Quigley G, Dison P, Pendray M. Selenium deficiency in low birth weight neonates: are unrecognized problem. J Pediatr. 1989;114:865-70.

35. Amin S, Chen SY, Collipp PJ, Castro MN, Maddalaiah VT, Klein SW. Selenium in premature infants. Nutr Metab. 1980;24:331-40.

36. Darlow BA, Winterbourn CC, Irider TE, Graham PJ, Harding JE, Weston PJ. The effect of selenium supplementation on outcome in very low birth weight infants: a randomized controlled trial. The New Zeland Neonatal Study Group. J Pediatr. 2000;136: 473-80.

37. Darlow BA, Austin NC. Selenium supplementation to prevent short-term morbidity in preterm neonates. Cochrane Database Syst Rev. 2003;(4):CD003312.

38. Reifen RM, Zlotkin S. Microminerals. In: Tsang RC, Lucas A, Uauy $R$, Zlotkni $S$, editors. Nutritional needs of the preterm infant. Scientific Basis and Practical Guidelines. Baltimore: Williams \& Wilkins; 1993. p. 195-207.

Correspondência:

Cleide Enoir Petean Trindade

Rodovia Domingos Sartori, $\mathrm{km} 01$

CEP 18607-741 - Botucatu, SP

Tels.: (14) 3811.6274 / 3882.1472

Fax: (14) 3811.6274

E-mail: pediatri@fmb.unes.br 